The following is a brief résumé of the course of the disease obtained from the Fever Hospital notes: "On admission to the Homerton Fever Hospital the lesion was seen to be limited to the left iliac region and upper part of the thigh, extending from the upper limit of that region to a line parallel to Poupart's ligament and two inches below it. The ulceration was superficial and sinuous in outline. There were two deep ulcers in the inguinal fold and the left labium majus was swollen, red, and tense. There were no vaginal discharoe and no fotor. There were a few scattered ulcers about the size of split-peas in Scarpa's triangle below the general mass of ulceration. The child was pale ; pulse regular and of good size and tension; temperature $97^{\circ} \mathrm{F}$.; fauces pale and clean. 12,000 units of antitoxin were given and local treatment was applied. Cultures taken from the lesion showed the bacillus of diphtheria growing quite pure. The bacilli were mainly of the long variety. Oct. 1st: The child vomited twice during the night and several times during the day. She was therefore fed per rectum and the vomiting ceased. Oct. 4th: Vomiting again set in. The local lesion was rapidly healing. Oct. 5th: The vomiting was profuse and persistent and the urine was loaded with albumin. The wound was clean and granulating. From Oct. 5th to 18th: The child vomited persistently. Oct. 28 th : The patient was very pale and losing flesh rapidly. There was no obvious palsy but the child was very limp and helpless. Oct. 30th : Face without expression. Breathing jerky and irregular. No obvious palsy. Knee-jerks not obtainable. On Nov. 1st the following note was made: 'The child is lying in an absolutely helpless state, unable to move its legs which remain in whatever position they are placed. The arms, hands, and fingers can be moved slightly. All the muscles are flaccid and wasted. The knee-jerks cannot be obtained and the superficial reflexes are absent. The child can swallow. The respirations are jerky, with sucking in of the abdomen and intercostal spaces, suggesting paralysis of the diaphragm. The pulse is 168 , dropping a beat every now and then. The wound looks healthy but has not quite healed.' The child died at 10 P.M. on the same day (the forty-first day of the disease)."

Pathological examination.-A post-mortem examination was performed on the morning after death. The local lesion consisted of a patch of healthy granulation tissue about threequarters of a square inch in extent and surrounding this was a narrow zone of thin very slightly contracting cicatricial tissue. The scarring, indeed, suggested that the lesion had been much more superficial than its appearance at the beginning had suggested. The smaller ulcers had left no scars. The body was thin and emaciated. All the vital organs appeared healthy to the naked eye.

Histological report.-The heart was fixed in Müller's fluid and stained by Marchi's method. It showed a small amount of fatty degeneration comparable in extent with that found in a case of diphtheria proving fatal at this stage of the disease. There were no increase in the connective tissue of the heart and no intermuscular infiltration. The heart failure in this case was secondary. The amount of degeneration would probably not have given rise to any symptoms of heart failure except as the result of some strain. This is the usual course of events in diphtheria, death from heart failure in the later stages of that disease being secondary to a general failure of the nutritive processes, to some complication, or to some strain which the weakened heart is unprepared to meet. Extensive fatty degeneration was present in the liver but in no way to be compared with the almost total degeneration of the parenchyma which occurs in many fatal cases of late vomiting in diphtheria. Beyond slight fatty degeneration of the epithelium in the tubules the kidneys were normal. The lower part of the pons, the medulla, and the cervical and lumbar enlargements of the cord were examined by Nissl's method of staining. Here and there was seen a nerve cell of the multipolar variety in a condition of swelling and chromatolysis, but these were very rare when compared with the vast majority which were normal. On the whole, each region of the central nervous system examined was regarded as practically normal. The vagi, phrenics, median, intercostal, and anterior crural nerves were examined. All these nerves were normal except the anterior crural and here there was degeneration. The degeneration was in an early stage and although quite definite was not very extensive. Perhaps not more than about 10 per cent. of the fibres showed definite changes and not more than 1 per cent. were totally degenerated. The degeneration was more or less patchy in character, for the most part occupying but a small portion of the visible fibre. The changes consisted of cloudy swelling, the fibres being irregular in outline and finely granular in appearance. Many fat globules were seen and in these cases the myelin sheaths were completely broken up. In many fibres the axis cylinders were irregular in outline, in some places swollen and in others broken across. The majority of the degenerated fibres were lying singly in the middle of bundles of normal nerve fibres.

As mentioned at the beginning of the paper this case is interesting from the point of view of the rarity of this type of the disease nowadays and important in that it might easily have been undiagnosed except for a prompt bacteriological examination. As far as we are aware this is the first case which has been examined histologically and the findings agree with those which might have been expected to occur in a case of diphtheria proving fatal at a late period of the disease. The results of the pathological examination also afford confirmation of the fact that in the later stages of diphtheria very little fatty degeneration of the heart or degeneration of the cells of the central nervous system is to be found but that degeneration of the peripheral nerves is quite likely to be met with, whereas in cases dying from acute diphtheritic toxæmia extensive fatty degeneration of the heart and acute degeneration in certain cells of the central nerrous system are the rule. This question has recently been discussed by one of us (C. B.) in THE LANCET ${ }^{6}$ and therefore will not be entered into in this paper. Whether this was a case of primary cutaneous diphtheria or a diphtheritic infection of a previous lesion must remain an open question.

\section{TUMOUR OF THE RIGHT CAUDATE NUCLEUS AND FRONTAL LOBE.}

BY D. MCCAY, M.B., B.CH. R.U.I.,

CAPTAIY, I.M.S.; RESIDENT PHYSICIAN, MEDICAL COLLEGE FOSPITA CALCUTTA ;

AND

E. O. THURSTON, F.R.C.S. ENG.,

CaptaIN, I.M.s.; Resident SURGEON, MEDICAL COLLEge hospital, CALCUTTA.

THE following case of a glio-sarcoma of the caudate nucleus and prefrontal lobe of the right side appears to us to be of sufficient clinical interest to be put on record, not only on account of the difficulty of arriving at a definite diagnosis but also on account of the peculiar set of signs and symptoms exhibited by the patient. The degree of difficulty in the localisation of tumours of the cerebrum varies with the site, and the difficulty is very much greater if the tumour should be situated in those parts of the brain to which the name of "silent areas" has been applied. The prefrontal lobe has generally been considered to be one of these areas. It has been pointed out by many observers that a lesion of the frontal lobe does give rise to a fairly well-defined group of symptoms. In a most interesting and instructive paper in THE LANCET of Feb. 8th, 1902, p. 363, Dr. W. Elder and Mr. A. Miles have recorded a case of tumour of the left prefrontal lobe successfully removed by operation and they have fully discussed the diagnosis and symptoms observed in their case. The perusal of this paper gave great assistance in arriving at an opinion of the true nature of the condition of our patient. The symptoms observed in lesions of the frontal lobe they consider to be produced by " an interference with inhibition, with attention, and with judgment, which are probably the highest functions of the brain, rendering man pre-eminent amongst living creatures."

The patient, a man, aged 35 years, was an engineer in charge of a steamer on the voyage from England to Calcutta. All the information obtainable was that on the way out he appeared to be very forgetful, careless regarding the performance of his duties, and that all his desires were covered by the two words "food" and "sleep." There was no history of syphilis, alcohol, or mental troubles. On the arrival of the ship in Calcutta on Feb. 20th, 1904, the patient was admitted to the General Hospital for pain in the right side of the head and fits of giddiness. He was found to have some loss 
of memory, particularly with regard to recent events, and to have lost in a great measure all sense of decency and shame. While in hospital he improved to some extent and left to rejoin his ship, but was taken ill immediately again and was brought to the Medical College Hospital and admitted on April 5th, 1904.

On admission the patient, who was a strong, powerfully built man, was very dull and slept practically all the time. He could be roused easily enough but was very slow in answering questions and evidently could not keep his attention fixed on any one subject for any length of time. On being left alone he immediately went to sleep again. On being questioned as to where he was he always replied "in the General Hospital," although repeatedly informed that he was in the Medical College Hospital. Hearing was normal ; there was no aphasia and cerebration appeared to be slow and deliberate. Reading and writing could not be tested, as he refused to oblige us. There was paresis of the lower part of the face on the left side and of the left arm and leg; there were no sensory symptoms. The reflexes were lost; there were no ankle clonus and no trophic disturbance. The patient could only walk a few steps and then by throwing most of the weight of the body on the right side ; he was distinctly unsteady in his movements and he showed a great tendency to fall to the right when sitting up in bed or on attempting to walk. He complained of hearache all the time, which was limited to the frontal region of the right side. The skull over the right frontal lobe was painful and tender on percussion and appeared to be slightly swollen. There were no real involvement of the sphincters of the rectum and bladder, no vomiting, and no optic neuritis ; the bowels were constipated. He still took a marked interest in his food, though listless and apathetic to a marked degree concerning everything else. With regard to the circulatory system the heart was normal and the pulse was slow and regular and of moderate tension; there were no signs of atheroma. The temperature was $98^{\circ} \mathrm{F}$. and continued normal till after the operation. The other systems were normal.

As to the progress of the case there was a steady increase in the dulness and apathy and on April 8th he passed urine and fæces in the bed, probably not from any real loss of control of the sphincters but from carelessness regarding the impropriety of doing so. On the 12th his bowels were moved with difficulty and he began to have some trouble in swallowing. By the 19th the lethargy passed into stupor and he could only be awakened with great difficulty; all desire for food also seemed to have gone. The paresis did not become any more marked and there was never any real paralysis. The tenderness and bogginess over the right frontal region increased and he always winced on percussion over it. The chief points on which we had to rely for a diagnosis were headache, giddiness, loss of memory, loss of modesty and sense of decency, slight paresis of the left lower face, arm, and leg, listlessness and apathy, slowness of pulse, with persistent normal temperature, and tenderness over the right frontal region. Speaking generally a picture such as this points to increased intracranial pressure. The diagnosis had to exclude hæmorrhage, embolism and softening, dementia and atrophy of the brain, and abscess.

A. Against hæmorrhage we had: (1) the gradual development of the symptoms; (2) the loss of memory and mental symptoms developing first; and (3) absence of any associated lesions-e.g., albuminuria, high-tension pulse, hypertrophied left heart, atheroma, \&c.

$B$. Against softening due to embolism or thrombosis. Embolism is excluded for the same reasons as hæmorrhage and the absence of any endocarditis or septic condition still further supports its exclusion. Thrombosis may be excluded from the absence of any history of syphilis, starvation, malaria, no general paralysis of motion or sensation, and the age of the patient.

$C$. Dementia and gradual involution of the nervous system, beginning in the psychical centres, might have explained the case but the history of the case and the short duration of the symptoms were sufficient to exclude dementia.

$D$. Against abscess we had: (1) no evident source of infection-ear disease, pyæmia, \&c. ; (2) persistent slowness of the pulse with normal temperature ; and (3) no history of injury, \&c.

$E$. In favour of tumour we had : (1) the gradual onset and steady increase in severity of the symptoms, indicating a gradual increase in the intracranial pressure; (2) the persistent headache, slow cerebration, and lethargy; (3) the slowness of the pulse, with normal temperature; and (4) the paresis of the left side of the body. Against this view was the fact that some of the most important signs of tumour of the brain were absent, more particularly vomiting, optic neuritis, and loss of reflexes, in what was considered a esion of the upper motor segment.

With regard to the locality we had the following localising points : (1) paresis of the left lower face, arm, and leg; (2) the peculiar mental symptoms; (3) headache chiefly confined to the right frontal region; and (4) tenderness and bogginess of the same region. The paresis pointed to an implication of the fibres passing from the Rolandic area to the internal capsule, and as the paresis was very imperfectly developed we considered that the pressure of the tumour backwards was limited. That the cortex was not involved was evidenced by the fact that there were no convulsions, no aura, and no epilepsy. The peculiar mental symptomsloss of memory, apathy, loss of sense of shame, loss of inhibitory power - we thought to be strong evidence of the lesion being situated in the frontal lobe. All these symptoms are intimately associated with abolition of the functions of the frontal lobe and are largely due to a loss in the power of inhibition of the individual, so that the patient is reduced almost to the condition of an animal deprived of its cerebrum. These points are extremely interesting in our case, as from a perusal of the literature of the subject one would be inclined to consider the left prefrontal region of more importance than the right as far as the above functions are concerned. (At the time we took pains to make sure that the patient was right-handed.) Allan Starr, from a study of the cases recorded of this region, concludes: "The form of mental disturbance in lesions of the frontal lobe does not conform to any type of insanity. It is rather to be described as a loss of self-control and a subsequent change of character. The mind exercises a constant inhibitory influence upon all action, physical and mental, from the simple restraint on the lower reflexes, such as the sphincters, to the highest control over the complex reflexes, such as emotional impulses and their manifestation in speech and expression. This action of control involves judgment and reason-the highest mental qualities. Thus we would expect the partial destruction of the frontal lobes to be accompanied by errors of judgment and reason of a striking character; one of the first manifestations would be a loss of that self-control which is the constant accompaniment of mental action and which would be shown by an inability to fix the attention to follow a continuous train of thought or to conduct intellectual processes. It was this very symptom that was present in one-half of the cases collected. It did not occur in lesions of any other part of the brain."

The absence of vomiting in our case may be due to the fact that the tumour was situated so far forward; as the nearer the tumour is to the vomiting centre in the medulla the greater the tendency to vomit. Our patient was examined for optic neuritis on admission, before the increase in intracranial pressure was marked, which may account for its absence. On the above evidence a definite diagnosis of tumour of the frontal lobe of the right side was made and the patient was transferred to the surgical side with a view to operation. On April 21st Captain Thurston opened the skull over the area of the tenderness; further than an evident increase in the intracranial pressure nothing abnormal appeared on the outer surface of the brain. An incision was made in the cortex and a finger inserted and the brain around was gently and carefully palpated for any mass but nothing was found. Although nothing could be discovered during the operation of the nature of a tumour, the diminution of the intracranial pressure alleviated the more pressing symptoms. Consciousness returned and the patient could answer questions and he made marked use of his arms, wiping his mouth with the sheet after coughing. He still passed urine and fæces in bed. Next day there was a good deal of coughing and expectoration. He tried to undo the bandages. $\mathrm{He}$ vomited and the temperature rose to $101 \cdot 5^{\circ}$. On the 24 th he became unconscious; his temperature was $103.2^{\circ}$. He never recovered consciousness and died on the evening of the 26th.

Necropsy. - At the post-mortem examination the following was the condition of the brain. 1. The right ventricle was distended with fluid and dilated anteriorly, bulging forwards into the frontal lobe. 2. The floor of the right ventricle showed a reddish-brown tumour of the corpus striatum, projecting into the cavity of the ventricle and pushing the third ventricle over towards the left. 3. The left ventricle contained excess of cerebro-spinal fluid. Nothing else 
abnormal was found. The tumour was a glio-sarcoma of the caudate nucleus, rapidly growing and projecting into the frontal lobe, pressing on and infiltrating the bundles of fibres coming from the frontal lobe to the internal capsule. The posterior part of the tumour was pressing on the internal capsule and would thus account for the motor paresis. 'The greatly increased amount of cerebro-spinal fluid with the accompanying increase in intracranial pressure explained the gradually increasing stupor. The several connexions of the frontal lobe with other parts of the brain would assist in explaining the mental condition of our patient. Our reason for recording this case is to add another to the list of lesions of this region already reported, where it has been possible to localise the site of the lesion before death.

\section{TWO CASES OF TETANUS TREATED BY ANTITETANIC SERUM.}

BY R. ATKINSON STONEY, M.B., B.CH., B.A.O. DUB. VISITING SURGEON TO THE ROYAL CITY OF DUHLIN HOSPILAL.

As the serum treatment of tetanus is still on its trial I think that no apology is needed for the following brief records of two cases which have recently been under my care in the Royal City of Dublin Hospital.

CASE 1.-The patient, a male, aged 34 jears, was admitted to hospital on Oct. 31st, 1904, suffering from severe burns of both hands and legs, the result of his bed taking fire from the ashes of his pipe while he was asleep. He was found by his wife unconscious in bed with the bedclothes smouldering. Both hands and wrists were severely burned, three fingers on the right and two fingers on the left hand being completely destroyed. The burns on the lower extremities were less severe and were situated chiefly around the ankles and heels. After admission the temperature rose gradually and reached $103.6^{\circ} \mathrm{F}$. on the fourth evening; it fell to $100^{\circ}$ by the seventh morning and remained between $99^{\circ}$ and $100^{\circ}$ for a week and the patient appeared to be doing well. The temperature 10 se to $101^{\circ}$ on the fourteenth evening but came down the next morning.

On Nov. 16th-that is, the seventeenth day after the accident-the patient complained of some stiffness of his neck. During the day this increased and in the evening there were some stiffness of the jaws and difficulty in opening the mouth fully. He was removed to the observation ward and powders containing 30 grammes of chloral hydrate and one drachm of bromide of potassium were ordered to be given by the rectum every six hours. On Nov. 17th the patient had frequent spasms during the day, chiefly affecting the abdominal muscles and lasting only two or three seconds; he was given a subcutaneous injection of ten cubic centimetres of Pasteur's antitetanic serum in the morning. He lay on his back with the knees drawn up, head and neck very rigid, and teeth clenched, but was able to take fluid nourishment well. The temperature at 4 P.M. was $96 \cdot 8^{\circ}$. He was very restless during the night and had very frequent spasms. While under observation he had 31 distinct spasmodic contractions of the abdominal muscles in two minutes; after this chloroform was administered to ind uce sleep. He slept only at short intervals for about four and a half hours. On the 18 th the temperature was $103 \cdot 4^{\circ}$. He had constant spasms. Ten cubic centimetres of antitetanic serum were injected into the spinal canal by lumbar puncture. The spasms became more severe and the patient died at 1 P.M., the temperature rising to $107^{\circ}$ an hour before death and remaining at that level till the fatal termination.

CASE 2.-A youth, aged 19 years, was admitted to hospital on the evening of March 9th, 1905, complaining of stiffness of the jaws and difficulty in swallowing. Three weeks before he got a splinter of wood under the nail of his right middle finger; it was removed at once and on admission the wound was completely healed and gave no pain. On Sunday, March 5th, probably the eighteenth day after the accident, the patient first noticed the stiffness which gradually became worse and pain appeared in the neck and shoulder. The bowels had not been opened since the previous Monday. He was unable to swallow anything except liquids. On examination risus sardonicus was well marked, he was unable to open his mouth more than half an inch, his tongue could only be protruded to a very slight extent, and marked contraction of the masseters, some opisthotonos, and contraction of the abdomin:l muscles were present. There were no stiffness or contraction of the muscles of the extremities and no spasms. 'The temperature on admission was normal and remained so throughout his stay in hospital.

The patient was ordered a large simple enema which had a very good result. He was ordered powders containing one drachm of bromide of potassium and half a drachm of chloral hydrate by the rectum every six hours. On March 10th he had slept for six and a half hours during the night. $\mathrm{He}$ felt better except for the stiffness of the neck which had increased. Ten cubic centimetres of Pasteur's antitetanic serum were injected subcutaneously. He passed a quiet day, he had no spasms, and his neck was less stiff in the evening. In the evening one slight spasm was observed while the patient was half asleep. On the 11th he had slept well during the night and was feeling better though his neck seemed stiffer. Ten cubic centimetres of antitetanic serum were injected. He slept a good deal during the day. On the 12 th he had slept all night. His neck was still very stiff. The abdominal muscles were very hard and well-marked opisthotonos was present. Ten cubic centimetres of antitetanic serum were injected. He was better able to open his mouth. On the 13th he had slept well all night. Ten cubic centimetres of antitetanic serum were injected. The patient was given some bread soaked in milk and beef-tea which he swallowed without difficulty. He was feeling much better, though the stiffness of the neck still continued and there was pain in the chest on drawing a deep breath. On the 14th he had slept all night. He had no pain in the chest, his neck was less stiff, and he was able to open his mouth better. The powders were ordered to be given every eight hours. From this time onwards the course of the case was uneventful, though the contraction of the abdominal muscles and the stiffness of the neck disappeared very slowly. On March 24th the powders were stopped and on the 31st the patient was allowed out into the grounds and was discharged on April 7th.

These two cases present a marked contrast to one another. In both the incubation period was long, in the first case 17 days and in the second case probably 18 days. In both the temperature was normal on the first appearance of the srmptoms, in the second case it remained normal throughout the course of the disease; but in the first case after a preliminary fall it rose rapidly through $10.2^{\circ}$ in 20 hours. In both cases the treatment was the samei.e., injection of serum and the administration of large doses of bromide of potassium and choral hydrate by the rectum. In the one case death occurred within 60 hours of the onset of the first symptoms whereas the second patient recovered. In the first case the spasms were both frequent and severe, while in the second only one was observed. Case 1 may, I think, be regarded as hyperacute in spite of its long incubation period and Case 2 was certainly chronic. The only conclusion that I can draw from these two cases is that the result was due to the degree of infection and was not materially altered by the treatment. Dublin.

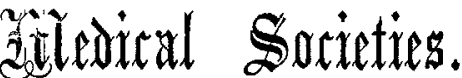

\section{PATHOLOGICAL SOCIETY OF LONDON.}

\section{Bacillus Influenze as a Cause of Endocarditis.-Gonococcu} Endocarditis.-The Vitality of the Typhoid Basillus in Shell-fish.

A MEETING of this society was held on April 18th, Dr. E. KLEIN, Vice-President, being in the chair.

Dr. T. J. HoRdER read a paper on the Bacillus Influenzæ as a Cause of Endocarditis and dealt with two cases of influenza septicæmia in which the bacillus was cultivated from the blood. The first case was that of a man, aged 31 years, who was admitted to St. Bartholomew's Hospital in May, 1904. Blood cultures were undertaken upon four separate occasions and were positive each time. Some of the cultivations obtained consisted of very large numbers of colonies, as many as 100 per cubic centimetre of blood. The method adopted was to puncture a vein with a sterilised needle attached to a five cubic centimetre glass syringe and to inoculate broth and agar tubes immediately. The morphological and cultural 